\title{
Editorial: Stimuli-Responsive Nanoparticles for Anti-cancer Therapy
}

\author{
Saji Uthaman ${ }^{1 *}$, In-Kyu Park ${ }^{2 *}$ Kang Moo Huh ${ }^{3 *}$, James Lai ${ }^{4 *}$ and Mitsuhiro Ebara ${ }^{5 *}$ \\ ${ }^{1}$ Chemical and Biological Engineering, IOWA State University, Ames, IA, United States, ${ }^{2}$ Department of Biomedical Sciences, \\ Chonnam National University, Gwangju, South Korea, ${ }^{3}$ Department of Polymer Science and Engineering, Chungnam National \\ University, Daejeon, South Korea, ${ }^{4}$ Department of Bioengineering, University of Washington, Washington, ME, United States, \\ ${ }^{5}$ International Center for Materials Nanoarchitectonic, National Institute for Materials Science, Tsukuba, Japan
}

Keywords: nanoparticle, drug delivery, photothermal therapy, molecular Imaging, ultra sound

Editorial on the Research Topic

Editorial: Stimuli-Responsive Nanoparticles for Anti-cancer Therapy

\section{OPEN ACCESS}

Edited and reviewed by: Gianni Ciofani,

Italian Institute of Technology (IIT), Italy

${ }^{*}$ Correspondence: Saji Uthaman suthaman@iastate.edu In-Kyu Park pik96@jnu.ac.kr Kang Moo Huh khuh@cnu.ac.kr James Lai

jilai@uw.edu Mitsuhiro Ebara EBARA.Mitsuhiro@nims.go.jp

Specialty section:

This article was submitted to Nanobiotechnology,

a section of the journal

Frontiers in Bioengineering and

Biotechnology

Received: 19 October 2021

Accepted: 21 October 2021

Published: 15 November 2021

Citation:

Uthaman S, Park I-K, Huh KM, Lai J and Ebara M (2021) Editorial: Stimuli-

Responsive Nanoparticles for Anticancer Therapy.

Front. Bioeng. Biotechnol. 9:797619.

doi: 10.3389/fbioe.2021.797619
The Research topic entitled "Stimuli-Responsive Nanoparticles for Anti-Cancer Therapy" addresses the current advances in the stimuli-responsive nanoparticles for anti-cancer. This issue comprises nine selected peer-reviewed manuscripts (original research and review articles) discussing the latest updates on various stimuli-responsive nanoparticles used in oncology. Different types of nanoparticles, including activated polymeric delivery systems (Peng et al.), multifunctional magnetic nanobubbles (Jin et al.), folic acid functionalized gelatin-AuNPs composite scaffolds (Chen et al.), Zinc oxide nanocrystals (Racca et al.), and Near-Infrared responsive Phase-shifting nanoparticles (Xu et al.) are presented through original research works. These novel nanoparticles with tailor-made properties offer a universal approach for anti-cancer therapy as their responsiveness depends on the general physiological properties commonly found in all tumors.

The first example of external stimuli-responsive nanoparticles is presented by (Racca et al.). In this study, amino-propyl functionalized $\mathrm{ZnO}$ nanocrystals ( $\mathrm{ZnO} \mathrm{NCs}$ ) combined with ultrasound shock waves (SW) were used to treat cancer cells. The $\mathrm{ZnO}$ NCs demonstrated synergism in combination with SW stimulus. In another study by Jin et al., multifunctional magnetic nanobubbles (MF-MNBs) comprising of poly (D, L-lactide-co-glycolide (PLGA) - polyethylene glycol-folate (PLGA-PEG-FA) polymer-based nanobubbles were evaluated as tumor-targeted ultrasound (US)/magnetic resonance (MR) imaging and focused ultrasound (FUS)-triggered drug delivery system. The MF-MNB exhibited ligand-receptor mediated tumor accumulation and focused ultrasound FUS-triggered drug delivery for efficient cancer treatment. Chen et al. have demonstrated photothermal ablation using near-infra-red irradiation as an external stimulus for killing cancer cells. This study synthesized folic acid (FA)-functionalized composite scaffold by hybridizing FA-conjugated gelatin and FAmodified AuNPs and using ice particulates as porogen material. In vitro and In vivo studies demonstrated that FA-functionalized gelatin-AuNPs composite scaffolds could elicit local photothermal ablation of breast cancer cells. Next, near-infrared responsive phase-shifted nanoparticles (NRPNs) have been designed by Xu et al. for magnetically targeted MR/US imaging and photothermal therapy of tumors. The near-infrared responsive phase-shifted nanoparticles (NRPNs) comprise PLGA nanoparticles encapsulated with indocyanine green (ICG), magnetic $\mathrm{Fe}_{3} \mathrm{O}_{4}$ nanoparticles, and perfluoro pentane (PFP). Upon irradiating with a NIR laser, the NRPNs, a phase-shifted expansion effect due to the quick conversion from light to heat by ICG and $\mathrm{Fe}_{3} \mathrm{O}_{4}$, can be used for ultrasound (US) imaging. In another study, Peng et al. have reported on an activated nanoparticle system comprising of poly (D, L-lactide-co-glycolide; PLGA)containing iron oxide nanoparticles (IOs) for biological imaging, using fucoidan/hyaluronic acid (FU/HA) to achieve targeting activity and applying polyethylene glycol-modified gelatin 
(PG)-carrying a phytochemical, epigallocatechin gallate (EGCG) to eradicate prostate tumors. This study demonstrated that the combination of therapeutic and molecular imaging could effectively target prostate cancer cells.

Besides the original research articles, this research topic also has a series of review articles that summarized the recent advances in anti-cancer therapy using external and internal stimuli-responsive metallic nanoparticles (Mohapatra et al.), pathological $\mathrm{pH}$-responsive polymeric nano biosensors (Kumar et al.), tumor microenvironment responsive nanoparticles (Thomas et al.), and plant virus nanoparticles (Hefferon et al. ). As a starting example, Kumar et al. have summarized the recent developments in the design, preparation, and characterization of $\mathrm{pH}$-responsive nanobiosensors and their ability to behave as efficient in vivo nano theranostics agents in acidic cancer environments. Thomas et al. have summarized the different types of internal ( $\mathrm{pH}$, redox, enzyme, ROS, hypoxia) stimuliresponsive nanoparticle drug delivery systems, Mohapatra et al., have outlined the role of different metallic nanotherapeutics in anti-cancer therapy, as well as their combinational effects with multiple stimuli for enhanced anti-cancer treatment. Finally, Hefferon et al. explore plant viruses as epitope-carrying nanoparticles and novel tools in cancer immunotherapy.

To summarize, we hope that this research topic will provide insights into the recent trends in nanomedicine, especially in oncology, using stimuli-responsive nanoparticles, providing insights into the development of targeted nanomedicine. The editors hope that the Research Topic "Stimuli-Responsive Nanoparticles for Anti-Cancer Therapy" will contribute to the progress of research and development activities in the field of nanomedicine, inspiring and offering a universal approach for anti-cancer therapy by taking advantage of the physiological properties commonly found in all tumors.

\section{AUTHOR CONTRIBUTIONS}

SU wrote the editorial, which was revised and proofed by IKP, and accepted by all the authors.

Conflict of Interest: The authors declare that the research was conducted in the absence of any commercial or financial relationships that could be construed as a potential conflict of interest.

Publisher's Note: All claims expressed in this article are solely those of the authors and do not necessarily represent those of their affiliated organizations, or those of the publisher, the editors and the reviewers. Any product that may be evaluated in this article, or claim that may be made by its manufacturer, is not guaranteed or endorsed by the publisher.

Copyright (c) 2021 Uthaman, Park, Huh, Lai and Ebara. This is an open-access article distributed under the terms of the Creative Commons Attribution License (CC $B Y)$. The use, distribution or reproduction in other forums is permitted, provided the original author(s) and the copyright owner(s) are credited and that the original publication in this journal is cited, in accordance with accepted academic practice. No use, distribution or reproduction is permitted which does not comply with these terms. 